\title{
Mise en œuvre de la DCE dans les zones littorales : préconisations pour le contrôle de surveillance et éléments pour le contrôle opérationnel
}

\author{
WFD implementation in coastal areas : technical recommendations for the \\ surveillance monitoring and technical elements for the operational monitoring
}

\author{
Anne Pellouin - Grouhel (*), Axel Romana (+) \\ (*) Ifremer Centre de Nantes rue de l'île d'Yeu BP 2110544311 Nantes cedex \\ e-mail : Anne.Grouhel@ifremer.fr
}

$\mathrm{(}^{+}$) Ifremer Centre de Toulon - zone portuaire de Brégaillon BP330 83507 La Seyne

The WFD monitoring requirements for surface waters were adapted to the coastal area with the selection of the relevant parameters to qualify the maritime ecosystems, with appropriate strategies of sampling.

Ifremer, and Cemagref for the fish indicator in the estuarine waters, are in charge of the elaboration of the monitoring programme in coastal waters (surveillance monitoring and operational monitoring). Ifremer has elaborated technical recommendations for the monitoring of the biological (benthic and phytoplanctonic) and chemical elements, according to many years of observations by the french monitoring networks (RNO, Rephy, Rinbio, RSL, Rebent).

These recommendations were established within experts groups. They clarify the parameters, the analytical methods, and the strategies of sampling for the classification of the water bodies in five ecological classes and two chemical classes.

The work on the relevant indicators of the ecological status still goes on, in relation with the 2006 european intercalibration exercise. Available data and future data will be used in order to set the borders between the classes of high, good, and moderate status, before the monitoring programme starts.

Data sets have to be completed to set the borders and to assess the ecological status of water bodies.

\section{INTRODUCTION}

Dans son annexe V, la directive cadre européenne sur l'eau identifie les éléments de qualité à prendre en compte pour chaque catégorie de masse d'eau afin d'établir leur état écologique. Les annexes VIII, IX et X (précisée par la décision 2455/2001/CE) fixent la liste des substances chimiques à prendre en considération dans la qualité globale des masses d'eau. Ces indications générales ont été déclinées localement pour définir le programme de surveillance qui sera applicable à partir de début 2007 pour le contrôle de surveillance et au plus tard en 2009 pour le contrôle opérationnel (contrôle des masses d'eau soumises à des pressions anthropiques telles qu'il y a un risque de non atteinte des objectifs écologiques en 2015).

Pour le contrôle de surveillance en zone littorale, les paramètres préconisés sont ceux qui sont pertinents pour qualifier les écosystèmes présents, à la fois les paramètres indicateurs de l'état de l'écosystème et les paramètres accompagnateurs nécessaires à l'interprétation, tels que la température, la salinité ou la turbidité.

Pour le contrôle opérationnel, les éléments de qualité proposés doivent permettre une évaluation de l'impact des pressions anthropiques et des changements d'état des masses d'eau concernées suite aux actions correctives entreprises à l'échelle du bassin versant. La liste des paramètres proposés, ainsi que les stratégies d'échantillonnage afférentes (fréquence des observations, localisation des points de prélèvement) dépend donc du type de contrôle et des conditions naturelles des masses d'eau, dont les caractéristiques physiques ont servi à déterminer des types.

La coordination technique du programme de surveillance des eaux littorales a été confiée à l'Ifremer par les acteurs national (Ministère de l'écologie et du développement durable) et locaux (agences de l'eau et directions régionales de l'environnement) chargés de l'application de la DCE sur le territoire français, ainsi qu'au Cemagref pour le suivi des populations de poissons dans les estuaires. 


\section{II $\square$ SURVEILLANCE DES PARAMETTRES BIOLOGIQUES ET DES PARAMÈTRES PHYSICO-CHIMIQUES}

\section{II.1 Surveillance des contaminants chimiques}

La surveillance des contaminants chimiques dans le milieu marin bénéficie de presque trente ans d'expérience en France, avec le RNO (réseau national d'observation de la qualité du milieu marin) piloté par l'Ifremer pour le compte du Ministère chargé de l'environnement. En 1998, l'agence de l'eau Rhône Méditerranée Corse a mis en place un complément à ce réseau sur les côtes méditerranéennes, le RINBIO (réseau intégrateur biologique), également opéré par l'Ifremer, pour la mesure des concentrations en contaminants chimiques dans des moules transplantées [Andral et al. 2004].

L'expérience acquise conduit à proposer de préférence un suivi chimique dans le sédiment fin [Pellouin - Grouhel coord. 2005] pour la plupart des substances identifiées par la DCE (substances qui s'adsorbent à la surface des particules sédimentaires : molécules hydrophobes et métaux), pour des raisons de représentativité de l'échantillon (caractère intégrateur du sédiment) et de performance des méthodes d'analyses. Les molécules retenues par les particules sédimentaires se concentrent sur le sédiment, et les méthodes d'analyses capables de détecter des niveaux de concentration de l'ordre du $\mu \mathrm{g}$ / $\mathrm{kg}$ (composés organiques) ou du $\mathrm{mg} / \mathrm{kg}$ (métaux) conviennent [Chiffoleau et al. 2003, Cossa et al. 2002, Tronczynski et al. 2005 (1)]. L'inertie de la contamination sédimentaire offre une bonne image de la qualité de la masse d'eau à l'échelle du plan de gestion, avec une évolution lente, fonction des vitesses de sédimentation et des remises en suspension. En revanche, pour le contrôle opérationnel qui demande une évaluation de l'évolution de la contamination d'une année sur l'autre, il est préconisé de rechercher ces mêmes molécules chaque année dans les organismes marins, témoins de la contamination ambiante [Chiffoleau et al. 2002 (2), Cossa et al. 2002, Munschy et al. 2005].

Pour les molécules exclusivement hydrophiles ( $\log \mathrm{K}_{\text {ow }}$ inférieur à 3) qui ne sont pas suivies par les réseaux de surveillance actuels, la recherche directement dans l'eau est incontournable, avec les méthodes appropriées [Chiffoleau et al. 2002 (1), Cossa et al. 2003, Michel \& Averty 2002, Tronczynski et al. 2005 (2)], à des niveaux de détection de traces et ultra-traces en rapport avec les normes de qualité environnementales (NQE) à venir. Dans un premier temps, ces molécules seront recherchées à la fréquence préconisée par la DCE (mensuelle), sur des points situés en amont et en aval des masses d'eau de transition. L'expérience ainsi acquise permettra par la suite de mieux cibler les prélèvements (fréquence, période). Autant que possible, les résultats de mesure seront complétés de résultats calculés à l'aide de modèles hydrodynamiques en accord avec les préconisations de Munschy et al. [2004] pour le suivi des phytosanitaires.

Les molécules volatiles qu'on ne retrouve pas dans le milieu marin sont à exclure de la liste des contaminants à rechercher.

\section{II.2 Surveillance des paramètres biologiques}

\section{II.2.1 Choix des paramètres pertinents}

La France métropolitaine est concernée par 2 régions bioclimatiques (ou écorégion) - le secteur Manche -
Atlantique et la Méditerranée - pour lesquelles il a été défini deux ensembles de types de masses d'eau distincts, avec des paramètres biologiques et des grilles de classification associées propres à chaque ensemble. Quant aux régions d'outre-mer (Antilles, Guyane et La Réunion), dont les eaux marines sont également concernées par l'application de la DCE, elles représentent un autre ensemble pour lequel les paramètres biologiques pertinents devront également être choisis.

Les groupes de paramètres sont pré-identifiés par la DCE (annexe $\mathrm{V}$ ) et la sélection finale des paramètres retenus dépend d'une part des caractéristiques des masses d'eau (typologie / habitats benthiques) et d'autre part des contrôles (surveillance ou opérationnel).

La prise en compte de la grande diversité des écosystèmes littoraux dans la sélection des paramètres a été assurée par la réunion de groupes d'experts français pour chaque groupe de paramètre. Cette méthode collégiale a permis de sélectionner une liste de paramètres adaptés à chaque élément de qualité biologique et aux objectifs de classification des masses d'eau. Quatre groupes d'experts, dont trois pilotés par l'Ifremer ont ainsi travaillé en 2004 et 2005 :

- groupe d'experts «poissons », piloté par le Cemagref (Bordeaux)

- groupe d'experts «phytoplancton », piloté par l'Ifremer (Nantes)

- groupe d'experts «végétation benthique », piloté par l'Ifremer (Brest)

- groupe d'experts « invertébrés benthiques », piloté par l'Ifremer (Brest).

Certains membres de ces groupes participent également aux groupes européens d'intercalibration, chargés de discuter les méthodes nationales d'évaluation, de manière à assurer une cohérence dans l'évaluation de la qualité biologique des masses d'eau au sein de la communauté des pays signataires de la DCE.

\section{II.2.2 Phytoplancton et paramètres physico-chiques explicatifs}

Parmi les éléments de qualité biologique pour la classification de l'état écologique, la directive cadre européenne sur l'eau identifie, pour les eaux côtières et de transition, la composition, l'abondance et la biomasse du phytoplancton. Les indicateurs retenus, issus de l'expérience acquise à travers vingt ans de surveillance du phytoplancton dans le cadre du Rephy (Réseau de surveillance du phytoplancton et des phycotoxines) sont pour ce paramètre [Pellouin - Grouhel coord. 2005] :

- la concentration en chlorophylle a (méthode décrite par Aminot \& Kerouel [2004]), pour la biomasse,

- le nombre annuel moyen d'efflorescences phytoplanctoniques de l'ensemble des espèces observées, pour l'abondance,

- le nombre annuel moyen d'efflorescences phytoplanctoniques des espèces nuisibles pour l'écosystème, pour la composition.

Dans les lagunes méditerranéennes, l'indicateur d'abondance est complété d'une estimation de l'abondance par classe de taille mesurée par cytométrie de flux [Vaquer et al., 1996 ; Gregori et al., 2001 ; Becker et al., 2002 ; Rutten et al., 2005]. Cette méthode permet de distinguer le picophytoplancton (environ $85 \%$ des cellules phytoplanctoniques) du nanophytoplancton [Jacquet et al.,1998]. 
En revanche, pour les eaux de transition de la zone macrotidale de la Manche et l'Atlantique, les estuaires sont plutôt des zones d'accumulation temporaire de la biomasse chlorophyllienne venue du bassin versant amont, et non pas des zones de production interne forte étant données les fortes turbidités; le suivi du phytoplancton en estuaire présente donc peu d'intérêt pour évaluer l'état écologique et il a été proposé de l'exclure des paramètres pertinents pour établir l'état écologique d'un estuaire dans cette zone géographique.

L'analyse de séries historiques longues montre une grande variabilité dans les eaux côtières d'une année sur l'autre, liée notamment aux facteurs climatiques et anthropiques. L'échantillonnage est donc réalisé tous les ans sans interruption sur la période du plan de gestion (six ans), pendant la période productive. Pour les eaux côtières de Manche et Atlantique, celle-ci s'étend de mars à octobre ; en revanche, elle est plus difficile à encadrer pour les eaux côtières méditerranéennes et un suivi tout au long de l'année est donc préconisé. Quant au suivi de la chlorophylle a dans les lagunes méditerranéennes, l'échantillonnage recommandé (période et fréquence) découle de l'analyse des données du RSL (Réseau de Suivi Lagunaire) qui a montré que les résultats acquis de juin à août donnaient une bonne image de la biomasse chlorophyllienne.

La directive cadre européenne sur l'eau prévoit également d'intégrer les paramètres physico-chimiques soutenant la biologie, dont les nutriments. Seul l'oxygène dissous présente un intérêt pour le contrôle de surveillance des eaux marines. La difficulté à établir un lien entre enrichissement en nutriments et eutrophisation, à cause du fort poids de facteurs naturels tels que l'hydrodynamisme de la zone, les matières en suspension qui limitent l'activité chlorophyllienne, la reminéralisation plus ou moins rapide de la matière organique détritique conduit à ne pas retenir les nutriments dans le contrôle de surveillance.

De plus le déphasage temporel entre le maximum de biomasse chlorophyllienne (généralement au printemps) et la concentration maximale de nutriments (en hiver) comme le montre la figure 1, accroît cette difficulté. Il est proposé de réserver les dosages des nutriments au contrôle opérationnel des masses d'eau sensibles à l'eutrophisation, en vue notamment d'évaluer l'efficacité des programmes de lutte contre les apports organiques dans ces zones.

\section{II.2.3 Faune et flore benthiques}

L'expérience française acquise dans le domaine des réseaux de surveillance des peuplements benthiques marins est plus limitée dans le temps et dans l'espace que pour les contaminants chimiques ou le phytoplancton mentionné précédemment. Les premiers enseignements tirés du réseau pilote Rebent Bretagne (réseau benthique) ont permis l'élaboration de fiches techniques pour chaque éléments de qualité retenus par le groupe d'experts pour le contrôle de surveillance [Guillaumont \& Gauthier, 2005]. En Manche - Atlantique, la surveillance se partage entre la zone intertidale (lorsqu'elle est suffisamment représentée dans la masse d'eau) et la zone subtidale pour le suivi concernant la flore benthique. Pour les invertébrés les experts internationaux ont considéré que c'était plus instable en intertidal, d'une part du fait des mouvements sédimentaires et d'autre part sur la suspicion d'une diversité moins forte en intertidal qui est un milieu plus sélectif (B. Guillaumont, comm. pers.) En Méditerranée, un nombre plus réduit d'élément de qualité a été retenu pour le contrôle de surveillance, sur une base essentiellement pragmatique (rapport information / coût). Les éléments de qualité benthiques retenus pour le contrôle de surveillance sont résumés dans le tableau 1.

\section{II.2.4 Réseau de surveillance DCE}

Le choix final des masses d'eau à suivre dans le cadre du contrôle de surveillance (fig. 2) a été fait dans chaque bassin, dans le cadre d'un groupe «littoral» rassemblant les experts techniques et les gestionnaires. Il a tenu compte d'une part de l'approche combinée des types et des catégories de pression exercées sur les masses d'eau (telles que définies dans l'état des lieux réalisé en 2004) et d'autre part de l'existence de points de mesure des réseaux existants, de manière à favoriser les séries chronologiques porteuses d'une information sur l'évolution des paramètres depuis plusieurs années. Pour les éléments de qualité benthiques, le suivi dépend des habitats présents. Or les données à ce sujet sont dispersées et inégales et devront être rapidement complétées par l'acquisition de nouvelles données pour pouvoir mettre en place un programme de surveillance cohérent.

Le programme de surveillance devra également être défini dans les départements d'outre-mer; le travail est en cours en Guadeloupe, Martinique, à la Réunion et en Guyane (information non disponible concernant Mayotte).

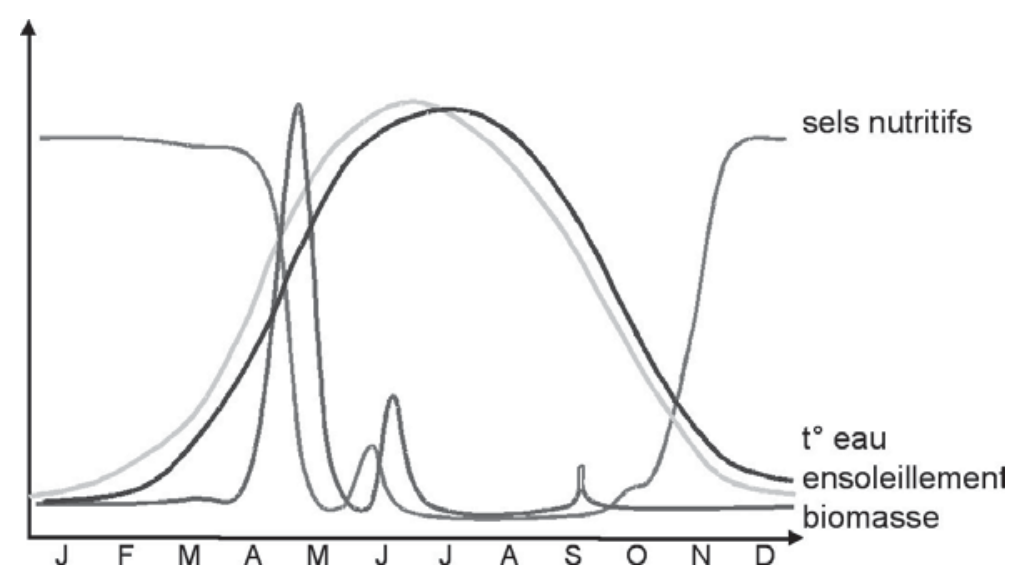

Figure 1 : Evolution annuelle du cycle des nutriments et de la biomasse phytoplanctonique dans les eaux marines [A. Daniel, note interne Ifremer]. 
Tableau 1. Synthèse des préconisations pour le suivi des peuplements benthiques marins [Guillaumont \& Gauthier, 2005].

\begin{tabular}{|c|c|c|c|c|c|}
\hline élément de qualité & paramètres & méthode & fréquence & période de suivi & localisation \\
\hline \multirow[t]{2}{*}{ maërl } & extension des bancs & $\begin{array}{l}\text { prospection acous- } \\
\text { tique }\end{array}$ & \multirow[t]{2}{*}{ tous les 6 ans } & \multirow[t]{2}{*}{ fin d'hiver } & \multirow[t]{2}{*}{$\begin{array}{l}\text { Cotentin ouest } \\
\text { Bretagne }\end{array}$} \\
\hline & état des bancs & $\%$ maërl vivant & & & \\
\hline \multirow[t]{2}{*}{$\begin{array}{l}\text { macroalgues inter- } \\
\text { tidales }\end{array}$} & $\begin{array}{l}\text { couverture } \\
\text { des fucales }\end{array}$ & télédétection & tous les 6 ans & juin - août & \multirow[t]{2}{*}{ Manche - Atlantique } \\
\hline & $\begin{array}{l}\text { composition } \\
\text { floristique }\end{array}$ & in situ & tous les 3 ans & avril - juin & \\
\hline \multirow[t]{2}{*}{$\begin{array}{l}\text { macroalgues subti- } \\
\text { dales }\end{array}$} & $\begin{array}{l}\text { Limite inférieure } \\
\text { de la ceinture de } \\
\text { laminaires }\end{array}$ & profondeur & \multirow[t]{2}{*}{ tous les 6 ans } & \multirow[t]{2}{*}{ juin - août } & \multirow[t]{2}{*}{$\begin{array}{l}\text { Manche - Atlantique } \\
\text { (surtout du Cotentin } \\
\text { à l'île d'Yeu) }\end{array}$} \\
\hline & vitalité & $\begin{array}{l}\% \text { de recouvre- } \\
\text { ment (plongée) }\end{array}$ & & & \\
\hline \multirow[t]{2}{*}{ herbiers à zostères } & suivi surfacique & télédétection & tous les 6 ans & \multirow{2}{*}{$\begin{array}{l}\text { fin printemps } \\
\text { - début d'été }\end{array}$} & \multirow{2}{*}{$\begin{array}{l}\text { Cotentin ouest } \\
\text { Bretagne } \\
\text { Arcachon }\end{array}$} \\
\hline & $\begin{array}{l}\text { caractérisation } \\
\text { de la végétation }\end{array}$ & $\begin{array}{l}\text { in situ et prélève- } \\
\text { ments }\end{array}$ & tous les 3 ans & & \\
\hline \multirow[t]{2}{*}{ herbiers à posidonies } & $\begin{array}{l}\text { structure de } \\
\text { l'herbier }\end{array}$ & in situ & tous les 6 ans & \multirow[t]{2}{*}{ mars - juillet } & \multirow{2}{*}{$\begin{array}{l}\text { région PACA } \\
\text { Corse } \\
\text { Languedoc-Roussillon }\end{array}$} \\
\hline & \begin{tabular}{|l|} 
mesures \\
individuelles \\
\end{tabular} & $\begin{array}{l}\text { mesures en } \\
\text { laboratoire }\end{array}$ & tous les 3 ans & & \\
\hline $\begin{array}{l}\text { macrophytes des } \\
\text { lagunes }\end{array}$ & $\begin{array}{l}\text { État des } \\
\text { peuplements }\end{array}$ & $\begin{array}{l}\text { \% recouvrement } \\
\text { végétal } \\
\text { richesse spécifique }\end{array}$ & tous les 3 ans & mai - juin & $\begin{array}{l}\text { lagunes } \\
\text { méditerranéennes }\end{array}$ \\
\hline $\begin{array}{l}\text { invertébrés des eaux } \\
\text { côtières }\end{array}$ & $\begin{array}{l}\text { dénombrement } \\
\text { et biomasse }\end{array}$ & & tous les ans & $\begin{array}{l}\text { fin d'hiver \& fin } \\
\text { d'été }\end{array}$ & $\begin{array}{l}\text { Manche - Atlantique } \\
\text { Méditerranée }\end{array}$ \\
\hline $\begin{array}{l}\text { invertébrés } \\
\text { des lagunes }\end{array}$ & $\begin{array}{l}\text { composition, densité } \\
\text { et biomasse }\end{array}$ & & tous les 3 ans & juin & $\begin{array}{l}\text { lagunes } \\
\text { méditerranéennes }\end{array}$ \\
\hline
\end{tabular}

\section{E ÉVALUATION DE L'ÉTAT ÉCOLOGIQUE ET DE L'ÉTAT CHIMIQUE}

La réflexion sur les indicateurs de qualité a démarré parallèlement à l'élaboration du programme de surveillance, d'une part pour l'identification des paramètres pertinents (paramètres nécessaires et suffisants) et d'autre part pour la définition des conditions du «bon état» qui permet d'identifier les masses d'eau qui risquent de ne pas l'atteindre en 2015 (masses d'eau faisant l'objet du contrôle opérationnel).

L'approche en termes d'évaluation de la qualité est assez nouvelle pour le milieu marin pour lequel il y avait eu jusqu'à présent surtout une préoccupation d'observation des tendances (suivi chronologique des phénomènes).

\section{III.1 Evaluation de l'état chimique en deux classes}

A ce jour, les normes de qualité environnementales (NQE), à définir à partir de critères écotoxicologiques pour chaque substance dangereuse, et communes à l'ensemble des pays signataires de la directive cadre européenne sur l'eau, ne sont pas arrêtées. Elles fixeront, pour chaque famille de molécules (substances prioritaires et substances dangereuses), la limite entre le bon et le mauvais état chimique. Des valeurs provisoires circulent pour commentaire au sein de chaque état, définies par référence à des concentrations dans l'eau. Ceci pose le problème de l'interprétation des résul- tats déjà acquis ou à acquérir par la surveillance chimique dans les eaux littorales qui sont des concentrations dans le sédiment ou dans les organismes marins. Deux démarches ont été entreprises pour les eaux littorales, en vue de concilier l'établissement de NQE universelles et l'utilisation de matrice adaptée pour la surveillance chimique :

- la traduction des concentrations observées dans le sédiment ou le milieu marin en concentrations équivalentes dans l'eau (application du Technical Guidance Document européen);

- la modélisation de la bioaccumulation de contaminants chimiques par les organismes marins (travaux sur les métaux de S. Casas [2005], qui seront étendus aux contaminants organiques en 2006).

\section{III.2 Evaluation de l'état écologique en cinq classes}

Les travaux menés au niveau français participent à la réflexion menée en 2005 et 2006 dans le cadre des groupes d'intercalibration géographiques européens (groupe nord est Atlantique et groupe Méditerranée). Ces travaux portent sur les trois niveaux supérieurs : très bon état / bon état / mauvais état de la classification. Ils seront complétés des mesures et observations réalisées dans le cadre du réseau des sites référence qui doit se mettre en place pour les eaux côtières et les eaux de transition au cours de l'année 2006. Ce réseau servira à définir les conditions de référence (très bon état écologique) pour chaque type défini de masses d'eau. 


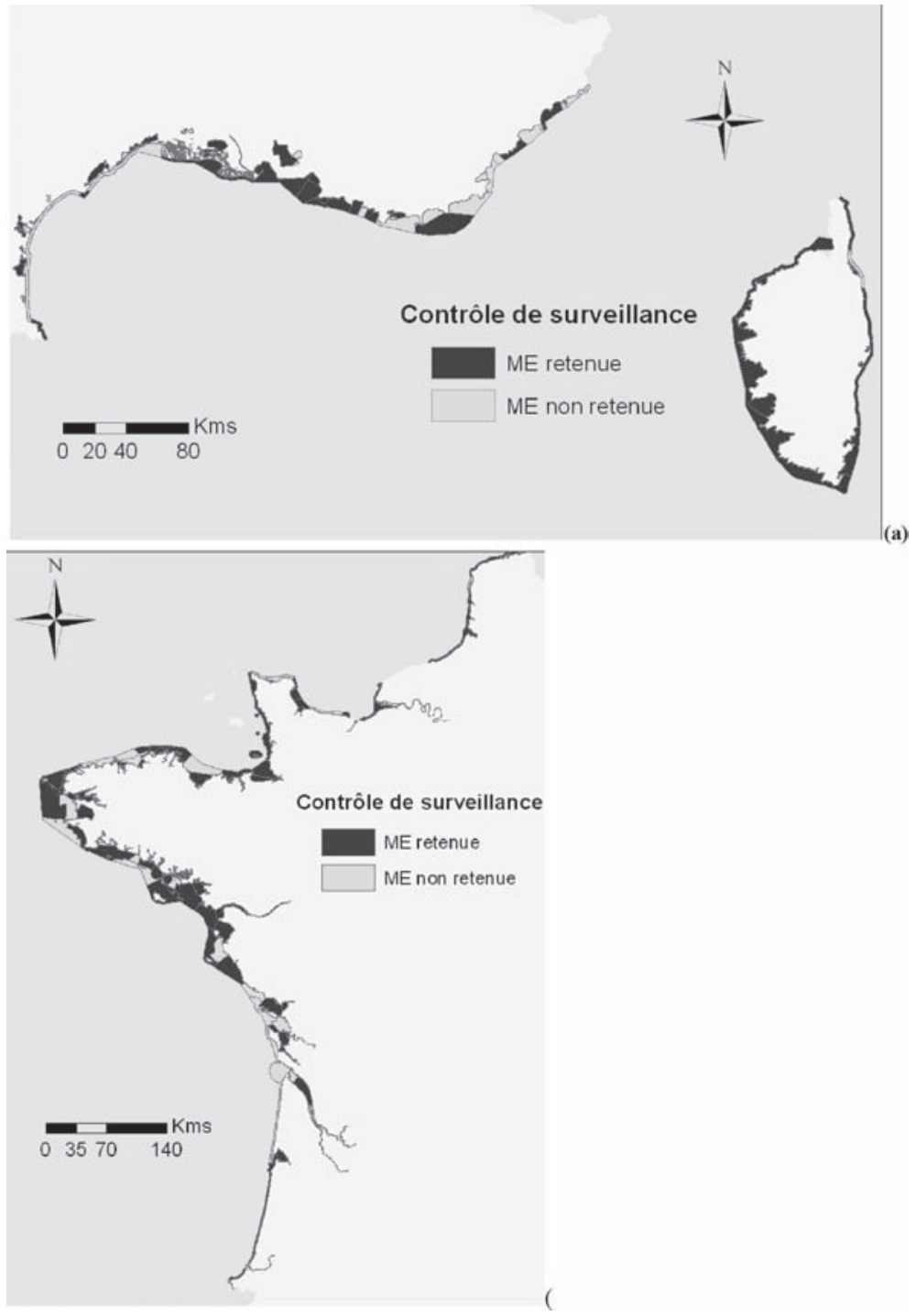

Figure 2 : masses d'eau littorales retenues pour le contrôle de surveillance (projet au 1/11/2005) (a) secteur Méditerranée et Corse (b) secteur Manche et Atlantique.

Pour l'indicateur de biomasse phytoplanctonique (percentile 90 de la teneur en chlorophylle a calculé sur une période de 6 ans) ainsi que l'indicateur physico-chimique associé (percentile 10 de la teneur en oxygène dissous calculé sur 6 ans), des grilles de lecture ont été établies à partir des données disponibles (tab. 2 et 3 ).
Pour les autres éléments de qualité biologiques, les travaux sont en cours. Différentes approches existent dans la littérature concernant le compartiment benthique. Elles devront être confrontées aux données obtenues sur un grand nombre de masses d'eau côtières pour en définir les conditions d'application.

Tableau 2. Etat écologique des masses d'eau: seuils pour l'indicateur de biomasse ( $P 90$ de la chlorophylle a) MEC : masses d'eau côtière.

\begin{tabular}{|l|c|c|c|c|c|c|c|}
\cline { 3 - 8 } \multicolumn{1}{c|}{ Façade } & Catégorie & $\begin{array}{c}\text { Période } \\
\text { prod. }\end{array}$ & $\begin{array}{c}\text { très bon } \\
\text { état }\end{array}$ & bon état & état moyen & $\begin{array}{c}\text { état } \\
\text { médiocre }\end{array}$ & $\begin{array}{c}\text { état } \\
\text { mauvais }\end{array}$ \\
\hline Manche/Atlantique & MEC & $03-10$ & $<5$ & $5-10$ & $10-20$ & $20-40$ & 40 \\
\hline $\begin{array}{l}\text { Méditerranée } \\
\text { (ouest Rhône) }\end{array}$ & MEC & $01-12$ & $<2$ & $2-4$ & $4-8$ & $8-16$ & $>16$ \\
\hline $\begin{array}{l}\text { Méditerranée } \\
\text { (est Rhône + Corse) }\end{array}$ & MEC & $01-12$ & $<1$ & $1-2$ & $2-4$ & $4-8$ & $>8$ \\
\hline Méditerranée & Lagunes & $06-08$ & $<5$ & $5-10$ & $10-20$ & $20-40$ & $>40$ \\
\hline
\end{tabular}


Tableau 3. Etat écologique des masses d'eau : seuils pour l'indicateur d'oxygène dissous (P 10).

\begin{tabular}{|l|c|c|c|c|c|}
\hline \multicolumn{1}{|c|}{ Seuils $\left(\mathbf{P}_{\mathbf{1 0}}\right)$} & très bon état & bon état & état moyen & état médiocre & état mauvais \\
\hline $\begin{array}{l}\text { Manche }- \\
\text { Atlantique } \\
\text { Méditerranée }\end{array}$ & $>5 \mathrm{mg} / \mathrm{l}$ & $5-3 \mathrm{mg} / \mathrm{l}$ & $3-2 \mathrm{mg} / \mathrm{l}$ & $2-1 \mathrm{mg} / 1$ & $\begin{array}{c}\leq 1 \mathrm{mg} / 1 \mathrm{ou} \\
\text { une valeur } \leq 1 \mathrm{mg} / 1\end{array}$ \\
\hline
\end{tabular}

Le manque de données pour les départements d'outre-mer ne permettra pas la proposition de grilles de lecture avant le démarrage des opérations de surveillance.

\section{GONCLUSIONS}

La mise en œuvre de la DCE sur le territoire français implique la mise en place de la surveillance adaptée à échéance de décembre 2006. Le programme de surveillance a été adapté au milieu marin au cours de l'année 2005 à partir de l'expérience acquise dans les réseaux de surveillance depuis plusieurs années. Pour les contaminants chimiques ceci a conduit à tenir compte, pour chaque famille de molécules, de la pertinence, du savoir-faire technique et des performances analytiques mobilisables. Pour les groupes de paramètres biologiques, la surveillance dépend de la typologie des masses d'eau et notamment des habitats benthiques présents, les lacunes dans les données benthiques devant être compensées dans les premières années du premier plan de gestion. Outre-mer, le manque de données sur l'environnement marin ainsi que le manque d'expérience en surveillance rend plus difficile l'élaboration d'un programme de surveillance adapté.

Un premier exercice d'évaluation de l'état des masses d'eau a été fait lors de l'état des lieux en 2004, à partir des données et connaissances disponibles. Il a montré les lacunes dans les données environnementales disponibles et le besoin de préciser les critères (grilles de lecture) qui seront appliqués à partir des données à acquérir. Ces données devront être de qualités homogènes et facilement accessibles pour les bilans («reporting») avec la nécessité d'un système complet et centralisé de gestion des données. L'Ifremer travaille actuellement à un nouveau système de bancarisation des données de l'environnement littoral - Quadrige.

De nouveaux outils pourraient être plus ou moins rapidement intégrés à la surveillance tels que l'exploitation des images satellites pour l'évaluation des niveaux de chlorophylle ou la mise au point de capteurs chimiques passifs.

\section{$V \square$ REMERCIEMENTS}

La synthèse des recommandations techniques pour une surveillance des eaux littorales adaptées à la DCE est le fruit du travail des coordonnateurs des différents réseaux animés par l'Ifremer (Rephy, RNO, RSL, Rebent Bretagne) qui se sont chargés chacun de réunir les avis des différents experts français sur le sujet. De nombreuses personnes ont donc été mises à contribution pour aboutir à ces conclusions, avec le soutien financier du Ministère de l'écologie et du développement durable / Direction de l'eau et des agences de l'eau chargées chacune de la mise en œuvre locale de la directive cadre européenne sur l'eau.
Tous nos remerciements vont également à Alice James (Ineris) pour le travail concernant les normes de qualité environnementales et l'état chimique, et à Cédric Fisson (Ifremer) qui a fourni durant toute l'année 2005 un indispensable soutien technique (cartographie, traitement de données, mise en forme de proposition de grilles ...) à l'ensemble de la réflexion pour la mise en œuvre de la DCE dans les eaux littorales.

\section{VI — RÉFÉRENCES ET CITATIONS}

Aminot A., Kerouel R. (2004). - Hydrologie des écosystèmes marins. Paramètres et analyses. Editions de l'Ifremer. Méthodes d'analyses en milieu marin. ISBN 2-84433-133-5

Andral B., Stanisière J.Y., Sauzade D., Damiera E., Thébault H., Galgani F., Boissery P. (2004). - Monitoring chemical contamination levels in the Mediterranean based on the use of mussel caging. Marine Pollution Bulletin 49 (9-10) : 704-712

Becker A., Armin M., Christian W. (2002). - Flow cytometric discrimination of various phycobilin-containing phytoplankton groups in a hypertrophic reservoir. Cytometry $48: 45-57$

Casas S. (2005). - Modélisation de la bioaccumulation de métaux traces $(\mathrm{Hg}, \mathrm{Cd}, \mathrm{Pb}, \mathrm{Cu}$ et $\mathrm{Zn})$ chez la moule, Mytilus galloprovincialis, en milieu méditerranéen. Thèse de doctorat, Université du Sud Toulon Var, Océanologie biologique et environnement marin

Chiffoleau J.F., Auger D., Boutier B., Rozuel E., Truquet I. (2003). - Dosage de certains métaux dans les sédiments et la matière en suspension par absorption atomique. Editions de l'Ifremer. Méthodes d'analyses en milieu marin. ISBN 284433-122-X

Chiffoleau J.F., Auger D., Chartier E., Grouhel A. (2002). - (2) Dosage de certains métaux traces $(\mathrm{Ag}, \mathrm{Cd}, \mathrm{Cu}, \mathrm{Cr}, \mathrm{Ni}, \mathrm{Pb}$, $\mathrm{V}, \mathrm{Zn}$ ) dans les organismes marins par absorption atomique. Editions de l'Ifremer. Méthodes d'analyses en milieu marin. ISBN 2-84433-114-9

Chiffoleau J.F., Auger D., Chartier E. (2002). - (1) Dosage de certains métaux traces dissous dans l'eau de mer par absorption atomique après extraction liquide-liquide. Editions de l'Ifremer. Méthodes d'analyses en milieu marin. ISBN 2-84433-104-1

Cossa D., Averty B., Bretaudeau J., Senard A.S. (2003). - Spéciation du mercure dissous dans les eaux marines. Dosage du mercure total, gazeux, réactif, mono et diméthylmercure. Editions de l'Ifremer. Méthodes d'analyses en milieu marin. ISBN 2-84433-125-4

Cossa D., Nakhlé K., Coquery M., Claisse D. (2002). - Dosage $\mathrm{du}$ mercure total et du monométhylmercure dans les organismes et les sédiments marins. Editions de l'Ifremer. Méthodes d'analyses en milieu marin. ISBN 2-84433-105-X

GrÉGORI G., Colosimo A., DenIS M. (2001). — Phytoplankton group dynamics in the Bay of Marseilles during a 2-year survey based on analytical flow cytometry. Cytometry $44: 247-256$ 
Guillaumont B., Gauthier E. (2005). - Recommandations pour un programme de surveillance adapté aux objectifs de la DCE. Recommandations concernant le benthos marin. Novembre 2005. Ifremer, rapport interne version 1 DYNECO/VIGIES 0511

Jacquet S., Lennon J.F., Marie D., Vaulot D. (1998). - Picoplankton population dynamics in coastal waters of the northwestern Mediterranean sea. Limnol. Oceanogr 43(8) : 1916-1931

Michel P., Averty B. (2002). - Analyse des composés organostanniques dans l'eau de mer. Editions de l'Ifremer. Méthodes d'analyses en milieu marin. ISBN 2-84433-108-4

Munschy C. (coordination), Arzul G., Bocquené G., Claisse D., Grossel H., Marchand M., Tissier C., Tixier C., TRONCZYNSKI J. (2004). - Stratégie pour la surveillance des produits phytosanitaires en milieu marin côtier. Rapport interne Ifremer R.INT.DEL-PC/2004.17

Munschy C., Tronczynski J., Héas-Moisan K., Guiot N., TRUQuet I. (2005). - Analyses des contaminants organiques (PCB, OCP, HAP) dans les organismes marins. Editions de l'Ifremer. Méthodes d'analyses en milieu marin. ISBN 284433-144-0
Pellouin - Grouhel A. (coordination). (2005). - Recommandations techniques pour un programme de surveillance adapté aux objectifs de la DCE. Stratégies d'échantillonnages et protocoles. Volume 1: contaminants chimiques, phytoplancton, hydrologie. Novembre 2005. Ifremer, rapport interne version 1 DYNECO/VIGIES 0512

Rutten TPA, Sandee B., Hofman, A.R.T. (2005). — Phytoplankton monitoring by high performance flow cytometry: A successful approach? Cytometry -PART-A. 64A (1) : 16-26

Tronczynski J., Munschy C., Héas-Moisan K., Guiot N., Truquet I. (2005). - (1) Analyses des contaminants organiques (PCB, OCP, HAP) dans les sédiments marins. Editions de l'Ifremer. Méthodes d'analyses en milieu marin. ISBN 2-84433-145-9

Tronczynski J., Munschy C., Héas-Moisan K., Guiot N., Truquet I. (2005). - (2) Analyses de contaminants organiques (herbicides, PCB, OCP, HAP) dans les eaux estuariennes et marines côtières. Editions de l'Ifremer. Méthodes d'analyses en milieu marin. ISBN 2-84433-148-3

Vaquer A., Troussellier M., Courties C., Bibent B. (1996). - Standing stock and dynamics of picophytoplankton in the Thau lagoon (northwest Mediterranean coast). Limnol. Oceanogr. 41 : 1821-1828 